\title{
Effect of Diabetes Mellitus on Sexual Arousal and Intercourse
}

\author{
${ }^{1}$ Department of Physiology and Biophysics, Stony Brook University School of Medicine, USA \\ ${ }^{2}$ Foley Plaza Medical, USA \\ ${ }^{3}$ Department of Urology, Stony Brook University School of Medicine, USA
}

Jason Gandhi ${ }^{1}$, Gautam Dagur ${ }^{1}$, Kelly Warren ${ }^{1}$, Noel Smith ${ }^{2}$ and Sardar Ali Khan ${ }^{1,3}$.

*Corresponding author: Sardar Ali Khan, M.D., F.A.C.S, F.R.C.S, Department of Urology, Health Sciences Center Level 9 Room 040, Stony Brook University School of Medicine, 101 Nicolls Road, Stony Brook, New York 11794-8093, USA; Tel: +1-631-987-0132, Fax: +1-631-444-7620; E-mail: skysalik@gmail.com

Received: Apr 20, 2016; Accepted: May 05, 2016; Published: May 10, 2016

Citation: Gandhi J, Dagur G, Warren K, et al. Effect of Diabetes Mellitus on Sexual Arousal and Intercourse. Transl Biomed. $2016,7: 2$.

\section{Abstract}

Diabetes mellitus, when producing hyperglycemia, as well as angiopathic, vasculopathic, and neuropathic complications, poses a threat to the function and viability of sexual arousal and intercourse at similar and different levels in males and females. Males are faced with hypogonadism, depression and anxiety, affecting their sexual arousal desire. Male intercourse may be impaired by erectile dysfunction, priapism, ejaculatory dysfunction, and/or benign prostatic hyperplasia. Female sexual arousal may be affected by depression, hormonal imbalance, and hypoactive sexual desire disorder. Female sexual intercourse may be disturbed by dyspareunia, vaginismus, and anorgasmia. Effects on sexual intercourse may also be seen at the gender neutral level due to cranial neuropathy and various autonomic neuropathies outside the genitourinary tract. Though specific treatments target most conditions, healthy diet and exercise are the best bets to avoid the long-term effects of diabetic complications on sexuality.

Keywords: Diabetes mellitus; Hypogonadism; Erectile dysfunction; Hypoactive sexual desire disorder; Cardiovascular autonomic neuropathy; Cranial nerve dysfunction

Abbreviations: Diabetes Mellitus (DM); Type 1 Diabetes Mellitus (T1DM); Type 2 Diabetes Mellitus (T2DM); Phosphodiesterase type 5 (PDE5); Cyclic Guanosine Monophosphate (cGMP)

\section{Introduction}

Diabetes mellitus (DM) is an increasingly common condition of chronic hyperglycemia and impaired carbohydrate, lipid, and protein metabolism, with over 550 million worldwide projected to be affected by 2030 [1]. Common risk factors for the development of DM include sedentary lifestyle, obesity, and increased caloric consumption. Type 1 (T1DM; insulindependent) and Type 2 (T2DM; noninsulin-dependent) DM can be major causes of system-wide micro and macrovascular complications that ultimately impede the ability to sexually arouse males and females, so that they may be able to adequately perform sexual intercourse.

Long-term effects of DM on the cardiovascular system are marked by accelerated atherosclerosis and hyaline arteriosclerosis (i.e. diabetic macroangiopathy) as well as specific capillary lesions (i.e. diabetic microangiopathy). Diabetic macroangiopathy increases incidence and severity of coronary artery disease, cerebrovascular accidents, peripheral ischemic arterial disease, and hypertension. Microangiopathy may develop several years after diabetes onset. Capillary lesions are associated with accretion of capillary basement membrane fragments, ultimately leading to capillary occlusion. Hypertension in diabetics may aggravate the progression of these macro- and microvascular complications [2-4]. Meanwhile, metabolically damaged nerve fibers, as well as thickening, hyalinization, and perivascular denervation of the vasa nervorum may be critical factors behind somatic, autonomic, and cranial nerve dysfunction (i.e. diabetic neuropathy) $[5,6]$.

This review utilizes a MEDLINE ${ }^{\circledR}$ database search to compile information on current pathogeneses and treatment options of sexual arousal and intercourse complications of DM. Most conditions below do not reflect a particular type of DM unless otherwise noted.

\section{Male Sexual Arousal Complications of Diabetes Mellitus}

\section{Hypogonadism}

Hypogonadism, or low levels of testosterone, may bring out symptoms such as decreased libido and energy. Depression and anxiety may also implicate the ability to attain sexual arousal [7]. The pathophysiological link between diabetes and hypogonadism is still poorly explained. Because obesity is often concomitant with diabetes, a possible hypothesis is that excessive estradiol secretion due to high aromatase activity in obese patients may inhibit gonadotropic releasing hormone secretion, thus decreasing testosterone secretion [8]. Another postulated cause is hypothalamic-pituitary-adrenal axis dysregulation-associated functional hypercortisolism [9]. 
Testosterone supplementation is the best way to treat this complication, and it is available through intramuscular injections, transdermal creams/gels, buccal tablets, and subcutaneous depots [7].

\section{Male Sexual Intercourse Complications of Diabetes Mellitus}

\section{Erectile dysfunction}

Erectile dysfunction is described as the inability to attain or sustain a normal erection despite appropriate sexual stimuli. Age and longer duration of diabetes are major risk factors [10]. It is reported to occur in more than half of diabetic men worldwide [11]. Vasculopathy, neuropathy, visceral adiposity, insulin resistance, and hypogonadism secondary to diabetes may be responsible for the pathogensis of erectile dysfunction [12]. Atherosclerosis and endothelial dysfunction may ultimately limit arterial blood flow necessary for erection to the penis [12]. Somatic neuropathy impairs sensory impulses from the penis to the reflexogenic erectile center, while autonomic neuropathy impairs parasympathetic relaxation of the corpus cavernosum smooth muscle [12]. Treatment for erectile dysfunction is primarily accomplished with oral phosphodiesterase type 5 (PDE5) inhibitors. Since the release of nitric oxide from cavernous nervous and endothelial cells is dwindled due to diabetic neuropathy and endothelial dysfunction, there is a lack of downstream upregulation of cyclic guanosine monophosphate (cGMP). cGMP relaxes penile smooth muscle for a proper erection. Because there is a shortage of cGMP, PDE5 inhibits the tendency of cGMP to break down and lose function [13]. Intracavernosal [14] or intraurethral [15] prostaglandin therapy, vacuum erection devices [16], and penile prostheses are some more involved solutions for sustaining erections. Diabetes may predispose penile implant recipients to infection [17]. Prothrombrotic factors secondary to diabetes may also be responsible for priapism, a chronic erection which may lead to painful sexual intercourse [18].

\section{Ejaculatory dysfunction}

Even though ejaculation is more of a concern for male fertility, it is also implicated in coitus as men report that the lack of ejaculation makes the act less pleasurable. Diabetic neuropathy may deteriorate the nerves controlling the external urethral sphincter. Because the sphincter remains open and urethral peristalsis is occurring to expel the ejaculate, the emission may end up in the bladder (i.e. retrograde ejaculation). Retrograde ejaculation may be treated with anticholinergics, antihistamines, and alpha-adrenergics. Premature ejaculation is the most prevalent of ejaculatory disorders. Anejaculation is the lack of ejaculation that may also present with the lack of orgasm, or anorgasmia [7].

\section{Benign prostatic hyperplasia}

Benign prostatic hyperplasia is described as the proliferative cellular growth of the glandular and stromal elements of the prostate. Diabetic vasculopathy and atherosclerosis may increase peripheral sympathetic nerve activity, causing hypoxia-related cell proliferation in the transitional zone of the prostate [19]. The resulting lower urinary tract symptoms are also concomitant with ejaculation difficulties and erectile dysfunction [20].

\section{Female Sexual Arousal Complications of Diabetes Mellitus}

\section{Depression}

Depression stemming from extensive diabetic complications may impair lifestyle, self-image, and relationship status, thus indirectly affecting sexual desire and arousal. In fact, psychosocial factors are likely the biggest reason women cannot achieve the proper sexual desire needed to enjoy intercourse [21].

\section{Hypoactive sexual desire disorder}

Hormonal imbalance regarding androgens, estrogens, and sex-hormone binding globulin [22] is a likely culprit for arousal disorder, which is defined by the lack of vasodilation and engorgement in the female external genetalia, namely the clitoris and vagina [23]. Diabetic neuropathy may be implicated in decreased innervation to the clitoris and vagina for blood flow, generating diffuse fibrosis of the clitoris and vaginal tissues, and thinning the musculature and epithelium of the vagina $[12,24,25]$. Endothelial dysfunction may be responsible for nitric oxide deficiency, which may be detrimental to clitoral smooth muscle [12]. Diabetic neuropathy may also be implicated in transducing sexual stimuli and the respective sexual response [12]. Atherosclerotic damage and endothelial dysfunction may also reduce vaginal lubrication and clitoral engorgement, causing decreased arousal [25].

\section{Female Sexual Intercourse Complications of Diabetes Mellitus}

\section{Dyspareunia}

Dyspareunia is described as painful sexual intercourse in women. Hyperglycemia from diabetes may reduce hydration levels of the mucous membranes of the vagina [12]. This lack of mucus lubrication can make it difficult and painful for the penis to slide effortlessly in during intercourse. The hyperglycemic state may also predispose the female to genitourinary infections- another possible cause for vaginal irritation-induced dyspareunia [12]. Atherosclerotic damage and endothelial dysfunction may also reduce vaginal lubrication and clitoral engorgement, inciting dyspareunia [25]. 


\section{Anorgasmia}

Dyspareunia is accompanied with a state of discomfort that makes it very difficult for the woman to enjoy sexual intercourse, thus putting sexual climax and the associated orgasm out of reach (i.e., anorgasmia).

\section{Gender Neutral Sexual Intercourse Complications of Diabetes Mellitus}

In addition to the gender-specific diabetic manifestations affecting optimal sexual arousal and performance, several factors may implicate the beginnings and the general course of coitus. Psychosocial factors such as adjustment to the diagnosis of DM, the burden of living with a chronic disease, and depression may impair sexual function, particularly in females [26]. Significant sensory, motor, and autonomic neuropathies of different physiological systems may affect proper sexual intercourse. Though extremely rare, diabetic cranial neuropathy involving cranial nerves III, IV, and VI may induce ophthalmoparesis, potentially implicating initial stages of sexual activity; retinopathy, optic neuropathy, and retinal vascular conditions may pose comparable threats [27].

DM may also present with cardiovascular autonomic neuropathy (CAN), giving rise to abnormalities in heart rate control and vascular dynamics [28]. This may elicit cardiac stress in strenuous physical exercise in this instance, sexual intercourse. CAN contributes to exercise intolerance by hindering response to change in heart rate, blood pressure, and cardiac output $[29,30]$. Dysfunction in parasympathetic and sympathetic responses results in exercise tolerance, which would otherwise improve cardiac output and increase perfusion to skeletal muscles [30]. Reduced ejection fraction, systolic dysfunction, decreased rate of diastolic filling, and blunted response to catecholamines may also limit exercise tolerance [30,31]. In cardiovascular disease-free diabetics exhibiting asymptomatic vagal CAN, exercise capacity (i.e., greatest tolerable workload and maximal oxygen uptake), heart rate, blood pressure, cardiac stroke volume, and hepatosplanchnic vascular resistance may all be diminished [30,32]. QT-interval prolongation, as well as cardio-autonomic nervous dysfunction, may contribute to the pathogenic mechanism of sudden unexpected death in diabetics [33]. The severity of CAN and heart rate are inversely proportional to one another during exercise [32].

The Health Professional's Guide to Diabetes and Exercise by the American Diabetes Association lists several more exercise risks secondary to diabetic autonomic neuropathy. Cardiorespiratory instability may pair with CAN. Defective sympathetic regulation and pseudomotor activity may lead to heat intolerance. Gastroparesis may delay delivery of nutrients to the bloodstream. Finally, there may be unawareness for hypoglycemic episodes [31]. Besides autonomic neuropathy, excess oxidative stress through the production of reactive oxidative species (e.g. superoxide anion, peroxide, and hydroxyl radicals) may also contribute to metabolic cardiac and skeletal muscle dysfunction, jeopardizing adequate sexual performance [34,35].

\section{Conclusions}

There has been a surge in patients exhibiting sexual intercourse and arousal complications as a reflection of the worldwide diabetes mellitus epidemic. Neuropathy, macroangiopathy, and microangiopathy due to increased oxidative stress and an immunodeficient state are the main malefactors behind the secondary conditions. Male diabetics may demonstrate erectile dysfunction, ejaculatory dysfunction, and hypogonadism. Females may exhibit hypogonadism, hypoactive sexual desire disorder, and dyspareunia. Female sexual dysfunction is still not as welldefined or pathophysiologically explained as male sexual dysfunction, although we assume there are many parallels. Cranial neuropathy and cardiovascular autonomic neuropathy may be implicated in sexual performance in either gender. Extensive study isolating the roles of gender, age, onset duration of diabetes, and diabetes as the individual risk factor (i.e. without the full aspects of metabolic syndrome) in the development of sexual complications remains to be adequately seen.

\section{Acknowledgements}

The authors are thankful to Drs. Kelly Warren, Todd Miller, and Peter Brink for departmental support, as well as Mrs. Wendy Isser and Ms. Grace Garey for literature retrieval.

\section{Conflicts of interest}

The authors declare no conflict of interest.

\section{References}

Federation ID (2013) IDF Diabetes Atlas, (6thedn) Federation ID (Eds) Brussels, Belgium.

Kim BH, Son SM (2006) Mechanism of developing diabetic vascular complication by oxidative stress. Journal of Korean Endocrine Society 21: 448-459.

Skrha J (2003) Pathogenesis of angiopathy in diabetes. Acta Diabetol 2: 324-349.

Orasanu G, Plutzky J (2009) The pathologic continuum of diabetic vascular disease. J Am Coll Cardiol 53: 35-42.

Duby JJ (2004) Diabetic neuropathy: an intensive review. Am J Health Syst Pharm 61: 160-173.

Nukada H (2014) Ischemia and diabetic neuropathy. Handb Clin Neurol 126: 469-487.

Lue TF (2000) Sexual Dysfunction in Diabetes, in Endotext, De Groot LJ, et al, Eds. South Dartmouth (MA).

Pitteloud N (2008) The relative role of gonadal sex steroids and gonadotropin-releasing hormone pulse frequency in the regulation of follicle-stimulating hormone secretion in men. $J$ Clin Endocrinol Metab 93: 2686-2692. 
9. Tirabassi G (2016) Diabetes Mellitus-Associated Functional Hypercortisolism Impairs Sexual Function in Male Late-Onset Hypogonadism. Horm Metab Res 48: 48-53.

10. Giugliano $F(2010)$ Determinants of erectile dysfunction in type 2 diabetes. Int J Impot Res 22: 204-209.

11. Thorve VS (2011) Diabetes-induced erectile dysfunction: epidemiology, pathophysiology and management. J Diabetes Complications 25: 129-136.

12. Maiorino MI, Bellastella G, Esposito K (201) Diabetes and sexual dysfunction: current perspectives. Diabetes Metab Syndr Obes 7: 95-105.

13. Dean RC, Lue TF (2005) Physiology of penile erection and pathophysiology of erectile dysfunction. Urol Clin North Am 32: 379-395.

14. Alexandre B (2007) Intracavernous injections of prostaglandin E1 for erectile dysfunction: patient satisfaction and quality of sex life on long-term treatment. J Sex Med 4: 426-431.

15. Garrido Abad P (2015) Safety and efficacy of intraurethral alprostadil in patients with erectile dysfunction refractory to treatment using phosphodiesterase- 5 inhibitors. Actas Urol Esp 39: 635-640.

16. Sun $L$ (2014) Combined sildenafil with vacuum erection device therapy in the management of diabetic men with erectile dysfunction after failure of first-line sildenafil monotherapy. Int J Urol 21: 1263-1267.

17. Christodoulidou M, Pearce I (2016) Infection of Penile Prostheses in Patients with Diabetes Mellitus. Surg Infect (Larchmt) 17: 2-8.

18. Sengupta N, Pandit K, Mukherjee S (2001) Priapism in type 2 diabetes mellitus. J Assoc Physicians India 49: 383.

19. Berger AP (2005) Vascular damage induced by type 2 diabetes mellitus as a risk factor for benign prostatic hyperplasia. Diabetologia 48: 784-789.

20. Bruskewitz RC (2003) Quality of life and sexual function in patients with benign prostatic hyperplasia. Rev Urol 5: 72-80.

21. Mazzilli R (2015) Sexual dysfunction in diabetic women: prevalence and differences in type 1 and type 2 diabetes mellitus. Diabetes Metab Syndr Obes 8: 97-101.
Feldhaus-Dahir M (2009) The causes and prevalence of hypoactive sexual desire disorder: part I. Urol Nurs 29: 259-260,263.

Yang CC (2006) Sexually responsive vascular tissue of the vulva. BJU Int 97: 766-772.

24. Kim NN (2006) Streptozotocin-induced diabetes in the rat is associated with changes in vaginal hemodynamics, morphology and biochemical markers. BMC Physiol 6: 4.

Park K (2002) Diabetes induced alteration of clitoral hemodynamics and structure in the rabbit. The Journal of urology 168: 1269-1272.

Giraldi A, Kristensen E (2010) Sexual dysfunction in women with diabetes mellitus. J Sex Res 47: 199-211.

27. Tu MC, Chang YY, Lin TK (2010) Recurrent multiple cranial neuropathies in a diabetic patient. Acta Neurol Taiwan 19: 208-212.

28. Schumer MP, Joyner S, Pfeifer M (1998) Cardiovascular autonomic neuropathy testing in patients with diabetes. Diabetes Spectrum 11: 227-231.

29. Kahn JK (1986) Decreased exercise heart rate and blood pressure response in diabetic subjects with cardiac autonomic neuropathy. Diabetes Care 9: 389-394.

0. Vinik Al, Erbas T, Casellini CM (2013) Diabetic cardiac autonomic neuropathy, inflammation and cardiovascular disease. Journal of diabetes investigation 4: 4-18.

1. Maser RE, Lenhard MJ (2005) Cardiovascular autonomic neuropathy due to diabetes mellitus: clinical manifestations, consequences, and treatment. The Journal of Clinical Endocrinology \& Metabolism 90: 5896-5903.

Vinik Al, Ziegler D (2007) Diabetic cardiovascular autonomic neuropathy. Circulation 115: 387-397.

Jermendy G (2003) Clinical consequences of cardiovascular autonomic neuropathy in diabetic patients. Acta Diabetol 40: 370-374.

34. Baynes JW (1991) Role of oxidative stress in development of complications in diabetes. Diabetes 40: 405-412.

5. Tsutsui H, Kinugawa S, Matsushima S (2011) Oxidative stress and heart failure. Am J Physiol Heart Circ Physiol 301: 2181-2190. 